\title{
PRESENTACIÓN. GERONTECNOLOGÍA Y EDUCACIÓN
}

\section{Presentation. Gerontechnology and education}

Antonio Víctor MarTín-García

Universidad de Salamanca

$\mathrm{D}$

ESDE DIVERSOS ÁMBITOS Y FOROS INTERNACIONALES se proclama la necesidad de construir la sociedad del s. xxi basada, entre otros, en la sostenibilidad, la inclusión y la calidad de vida para todos. Por ejemplo, en la Unión Europea (EU), la Estrategia Europa 2020 propone tres prioridades para los próximos años: crecimiento inteligente, crecimiento sostenible y crecimiento integrador. De hecho, presenta el Desafío Social bajo el lema «Europa en un mundo cambiante: sociedades inclusivas, innovadoras y reflexivas», y ello en el marco de realidades cada vez más tecnologizadas y envejecidas.

De un lado, y sobre la emergencia explosiva de una sociedad marcada por la presencia de diferentes tipos de tecnologías, basta señalar algunas cifras realmente espectaculares. Así, según reflejan los datos de We Are Social, en 2017 se ha producido un excepcional crecimiento de lo digital: más de la mitad de la población mundial utiliza internet, dos tercios de la población mundial (4.917 millones de personas) usan teléfono móvil, un tercio de la población mundial $(37 \%)$ son usuarios de redes sociales (2.549 millones de personas a través de teléfonos móviles). En España, según datos de 2017 tomados de la «Encuesta sobre equipamiento y uso de tecnologías de Información y Comunicación en los hogares» (INE, 20I7), el 83,4\% de los hogares españoles tiene acceso a la Red, el $84,6 \%$ de la población de 16 a 74 años ha usado internet en los tres últimos meses y el 69,0\% lo hace a diario.

De otro, los datos sobre la transformación demográfica no son menos contundentes, lo que explica la preocupación de políticos, economistas, etc., alarmados por las tendencias demográficas y su previsible impacto en los sistemas de salud y económico. Según datos del último informe recogido en el Global AgeWatch Index de 20I5, en ese año en el mundo había 9or millones de personas mayores de 60 años. Esa cifra alcanzará los 1.400 millones en 2030 (16,5\% de la población mundial) y representará el 21,5\% de la población mundial en 2050. Caso particular es Europa, donde se espera que más de la mitad de la población tendrá más de 65 años en 2050 (Eurostat, 2015). Hoy en España el I8,4\% de la población es mayor de 65 años (cerca de nueve millones de personas), y presentan una esperanza de vida entre las más altas de la Unión Europea (Abellan et al., 20I7). Quizá sea por este fenómeno poblacional que el enfoque de desarrollo humano del Programa de las Naciones Unidas para el Desarrollo (Agenda 2030 para el Desarrollo Sostenible); los Objetivos de Desarrollo del Milenio (ODM), 
que se ejecutaron hasta 20I5, o los nuevos Objetivos de Desarrollo Sostenible (oDs), más completos después de ese año, tratan el tema de envejecimiento de alguna forma en is de los 17 objetivos programáticos, aunque en algunos de ellos no se mencione explícitamente (por ejemplo, el Objetivo 3 propone «asegurar vidas saludables y promover el bienestar para todos a todas las edades» y el Objetivo 4 dice "Garantizar una educación inclusiva y equitativa de calidad y promover oportunidades de aprendizaje permanente para todos»), pero en todos estos objetivos se reconoce la importancia central de los hombres y las mujeres mayores para los ideales del desarrollo sostenible. De igual modo, la Organización Mundial de la Salud (oms), a partir del Informe mundial sobre el envejecimiento y la salud, aprobó «la Estrategia y plan de acción mundiales sobre el Envejecimiento y la Salud 20I6-2020», en la que se aboga por favorecer iniciativas de envejecimiento activo y saludable basadas en los principios de inversión social.

En la misma línea, el previsiblemente declarado Decenio del Envejecimiento Saludable (2020-2030) enfatiza este cambio conceptual que va de la idea de envejecer activamente a envejecer saludablemente bajo el lema «agregar salud a los años». Evidentemente, ambos aspectos, la actividad física y mental y la salud, están íntimamente relacionados y precisan adecuados entornos adaptados a las necesidades de las personas mayores. Pero el reto no se plantea en más actividad o más salud, sino en el mantenimiento de la capacidad funcional de las personas mayores cuanto más tiempo posible. En el Informe de la OMs de 2015 se entiende que «La capacidad funcional comprende los atributos relacionados con la salud que permiten a una persona ser y hacer lo que es importante para ella. Se compone de la capacidad intrínseca de la persona, las características del entorno que afectan esa capacidad y las interacciones entre la persona y esas características» (p. 30).

Por lo tanto, un aspecto que afecta a esa capacidad funcional tiene que ver con las características del entorno y el ajuste entre este y la persona. En la literatura especializada se utiliza cada vez más el concepto de «entorno amigable» para referir espacios adaptados, recursos y servicios accesibles que permiten maximizar la autonomía y las capacidades y las habilidades de las personas. Un ejemplo de esto puede verse en el deseo de los mayores de envejecer y vivir el mayor tiempo posible en su propia comunidad, en su casa (a esto se denomina «envejecer en el lugar» o «envejecer en la comunidad en la que se ha vivido»). Este deseo solo puede verse satisfecho a través de una investigación e innovación constante que promueva el desarrollo tecnológico que, a su vez, permita avanzar en soluciones asistenciales, sanitarias y de fomento de adecuadas interacciones sociales, como base para mantener el sentido de pertenencia, autonomía e integración social y, en definitiva, del bienestar y la calidad de vida de las personas en sociedades envejecidas.

Por esta razón, los grandes organismos internacionales abogan por el desarrollo tecnológico adaptado a las necesidades de determinados grupos sociales vulnerables, proclamando la necesidad de prestar una mayor atención a los efectos de la tecnología sobre aquellos grupos sociales que presentan más dificultades para el acceso y uso de las mismas y reclaman, en consecuencia, mayores esfuerzos políticos, sociales, económicos y de la comunidad científica internacional, para no solo mitigar esos efectos, sino también para impulsar avances tecnológicos que aseguren una mayor integración e inclusión social de las personas mayores y redunden, en definitiva, en mayores cotas de salud y de bienestar general. Por ejemplo, tanto en el Programa Grundtvig para 
el Aprendizaje a lo largo de la vida, como a través de Horizonte 2020, el Programa Marco de la UE para la Investigación y la Innovación, se recoge como tema transversal la investigación en Ciencias Sociales y Humanidades, entendiéndose las nuevas tecnologías como instrumentos para la solución de los problemas sociales y se destaca la necesidad de mejorar la calidad de la educación para evitar, entre otras cosas, la brecha digital entre usuarios y no usuarios. De igual modo, en España, el documento Marco de actuación para las personas mayores editado por el IMSERSO en 2014 recomendaba «Impulsar la innovación en las empresas para que desarrollen nuevos productos y servicios relacionados con las personas mayores».

Pues bien, ambos elementos, tecnologías y envejecimiento, o personas mayores, constituyen los ejes sobre los que pivota el presente monográfico de Aula. Revista de Pedagogía. En el fondo de este tema persiste la dialéctica entre quienes entienden que la rápida transformación de las sociedades actuales, como consecuencia de la omnipresencia de las tecnologías digitales, resulta un elemento perturbador y condicionante del desenvolvimiento funcional de este colectivo, y, al tiempo, la falta de acceso y uso de las mismas, un elemento que acrecienta su desigualdad social. Por el contrario, para otros, las soluciones tecnológicas resultan fundamentales para abordar las necesidades de una población altamente envejecida, imprescindibles para mitigar las enormes demandas y costos del sobreenvejecimiento en los sistemas de salud, económico y asistencial.

Todo esto explica la combinación de esfuerzos de distintas comunidades científicas y técnicas, con tradiciones y enfoques diferentes, en favor de objetivos comunes que, desde la idea del envejecimiento activo y saludable, enfatizan la necesidad de la mejora de la calidad de vida de las personas mayores a partir de desarrollos tecnológicos específicamente destinados para ellos. Un ejemplo claro de esto es el nacimiento y expansión de una nueva área de conocimiento en torno a la gerontecnología, una disciplina emergente que nace de la mezcla de la tecnología y el estudio del envejecimiento. La idea base de la gerontecnología es encontrar soluciones innovadoras basadas en tecnología para hacer frente a las complejas demandas de atención de este grupo de población. En suma, el objetivo de este campo de investigación e innovación es propiciar un enfoque multidisciplinar en el que participan áreas relacionadas con la Medicina, la Neurología, la Psicología, la Informática y Electrónica (Inteligencia Artificial), Ingeniería Biomédica y algunas más. Hasta ahora las principales líneas de desarrollo en el campo de la gerontecnología han estado relacionadas con aplicaciones clínicas, dispositivos de asistencia, tecnologías domésticas inteligentes, redes de comunicación, tecnología robótica, etc. A estos aportes queremos sumar la perspectiva pedagógica y educativa.

Sin embargo, esta aproximación pedagógica a este campo no está exenta de dificultades e incomprensión. Por eso nos planteamos algunos interrogantes, como son: ¿en qué medida el enfoque pedagógico y educativo puede contribuir con propuestas eficaces en este campo?; ¿cuál puede ser el papel de la pedagogía y de la educación?; ¿tiene sentido fomentar desde la educación recursos y dispositivos que introducen inevitablemente un componente de virtualidad en las relaciones entre personas (lo cual puede significar la pérdida de calidad en la relación física humana) desde el momento que estas acaban siendo mediadas por elementos tecnológicos?

Algunas de las respuestas a estas cuestiones tratamos de mostrarlas en este monográfico. Sin embargo, debemos asumir claramente que queda mucho camino por 
recorrer, los pasos dados hasta el momento en esta dirección son absolutamente insuficientes y queda claro que el acercamiento pedagógico a este problema es muy embrionario y apenas se ha iniciado. A pesar de ello, y en todo caso, defendemos aquí la idea de que la educación tiene mucho que aportar en este proceso tecnología-mayores fundamentalmente favoreciendo un uso inteligente de la tecnología: ya sea ayudando a personas mayores a conocer y utilizar dispositivos y recursos existentes como aportando enfoques educativos en el diseño y desarrollo de nuevos productos adaptados a sus necesidades. Es desde esta perspectiva desde la que presentamos cinco trabajos que ejemplifican diversos aspectos del acercamiento educativo al área de conocimiento interdisciplinar que ofrece la gerontecnología.

El primer artículo, presentado por el profesor de la Universidad de Salamanca Antonio Víctor Martín, busca ofrecer una visión general sobre las relaciones entre envejecimiento y tecnologías digitales y se presenta, en el marco de la gerontecnología, el papel que la pedagogía y la educación pueden tener en este nuevo ámbito multidisciplinar.

El segundo trabajo, presentado por Juan Carlos Aceros, profesor de la Universidad Industrial de Santander, Colombia, titulado «Robots para el cuidado de personas mayores. Taxonomía de una promesa», analiza los avances generales de la geronrobótica, un área de gran expansión en la actualidad para el desarrollo de soluciones tecnológicas pensadas como ayudas técnicas individuales y domésticas. En particular, se identifican algunas utilidades de este tipo de recursos, concretamente, los robots médicos, de servicio, sociales, recreativos, educativos, rehabilitadores o con potencial terapéutico.

El tercer artículo, firmado por José Miguel Toribio-Guzmán y varios miembros del Instituto Ibérico de Investigación en Psicociencias y la Fundación INTRAs, bajo el título «Rehabilitación cognitiva por ordenador en personas mayores: programa GRADIOR», presenta una línea de trabajo de gran interés como es la rehabilitación cognitiva por ordenador para la estimulación y mejora del funcionamiento cognitivo en personas mayores y describe uno de estos programas, GRADIOR 4.5 en su última versión, especificando sus diferentes componentes, metodología de aplicación y sus aspectos más relevantes.

Nuestro cuarto trabajo, realizado por las profesoras Roser Beneito-Montagut, Arantza Begueria y Nizaiá Cassián de la Universidad de Cardiff (Gales) y la Universidad Abierta de Cataluña (UOC), bajo el título «"Hago de todo y no sé hacer funcionar nada": aprendizaje afectivo y relacional de tecnologías digitales en adultos mayores», presenta un estudio desde la perspectiva de la etnografía digital en el que tratan de conocer cómo los adultos mayores usan y aprenden nuevas tecnologías en contextos no formales, cotidianos y relacionales, buscando reexaminar conceptos de aprendizaje y sus vínculos con la formación de identidades en torno a dos ejes: la edad y la tecnología, todo ello relacionado con el contexto de las relaciones y las redes sociales y el aprendizaje afectivo.

El último artículo que presentamos en el monográfico está realizado por Cristina Moreno, Cristina Alconada y José Dulac, vinculados a la Universidad Autónoma de Madrid y a los proyectos «Pluma y Arroba y Aula Adaptativa». En él se recoge una experiencia de formación en TIC con personas mayores centrada en el uso de recursos como son la pizarra digital y las tabletas para navegación por internet, así como otras aplicaciones como robots básicos para iniciar la programación y gafas de realidad virtual. 
PRESENTACIÓN

GERONTECNOLOGÍA Y EDUCACIÓN

\section{Bibliografía}

Abellán García, A.; Ayala García, A. y Pujol Rodriguez, R. (20I7) Un perfil de las personas mayores en España, 2017. Indicadores estadísticos básicos. Madrid, Informes Envejecimiento en red n. ${ }^{\circ}$ I5, 48 pp. [Fecha de publicación: 31/oI/2017; 27/03/20I7]. http://envejecimiento.csic.es/documentos/documentos/enred-indicadoresbasicosi7.pdf.

Asamblea Mundial de la Salud (2016) Acción multisectorial para un envejecimiento saludable basado en el ciclo de vida: proyecto de estrategia y plan de acción mundiales sobre el envejecimiento y la salud. Informe de la Secretaría. Declaración, oms, Ginebra, mayo, 2016.

Global overview \& We Are Social y Hootsuite (2017) Digital in 2017 Global Overviewa Collection of Internet, social media, and mobile data from around the world Digital in 2017, enero, 2017. http://bit.ly/GD20I7RO.

Inserso (20I4) Marco de actuación para las personas mayores. Madrid: Ministerio de Sanidad, Servicios Sociales e Igualdad.

Instituto Nacional de Estadística (2017) INEbase. http://www.ine.es/.

Organización Mundial de la Salud (oms) (2015) Informe Mundial sobre el Envejecimiento y la Salud. Ginebra, Suiza. Consultado en http://apps.who.int/iris/bitstream/ I0665/I86466/I/9789240694873_spa.pdf?ua=I.

Unión EUROPEA. Europa 2020: Una estrategia para un crecimiento inteligente, sostenible e integrador. http://eur-lex.europa.eu/legal-content/ES/TXT/?uri=celex:52010DC2020. 
